\title{
Activity Analysis of Albanian Speaking Facebook Accounts Spreading Violent Extremist Content
}

\author{
By Haris Fazliu
}

\section{Introduction}

Not all individuals that show online support for certain terrorist organisations will resort to violence, but all are at risk of becoming potential perpetrators of such acts. There are various statistics regarding participation of ethnic Albanian foreign fighters joining ISIS and Jabhat Al-Nusra in Syria and Iraq. The exact number of participants can not be confirmed but what we do know is that there are over 500 individuals that have participated in these conflicts ${ }^{1}$. Most of them have returned to the Balkans, some have died in the battlefields, and few are either captured or in camps. Imams and other individuals have also been charged and sentenced in Kosovo, Albania, and North Macedonia for financial and logistical support to ISIS and other Jihadi organisations. However, terrorist attacks are not prevalent in the aforementioned countries, and none have been successfully carried out. Nevertheless, terrorist organisations have continued to extend their reach to these regions through a persistent presence online and on social media.

\section{Methodology and Research Design}

The goal of this study was to identify Albanian speaking profiles on Facebook that support Islamic terrorist organisations and Islamic Violent Extremism (Jihadi) ideology and quantify their online activity. We chose to analyse Albanian speaking accounts on Facebook due to the platform's popularity in the region and the fact that various terrorist organisations use Facebook to target the Albanian speaking audience. ${ }^{2}$ Although these groups are known to use other platforms as well, such as Telegram, this study focuses exclusively on the Facebook presence.

\section{Sample Selection:}

Over a short period (March 31st and April 10th 2020) we identified and analysed social media accounts that openly support Islamic terrorist groups and Jihadi ideology. All the accounts are of Albanian-speaking individuals based mostly in the Western Balkan region. Using false personas and anonymous accounts we managed to detect certain Jihadi pages (using string searches) that spread ISIS and similar Jihadi propaganda. As initial accounts were identified, the snowball sampling method was then utilized. For instance, once an account was identified, the friends list was searched for similar accounts and likewise added to the sample. Each profile was analyzed, and information regarding each account was collected manually. Through this process we were able to identify 400 individual accounts supporting ISIS and other groups with similar ideologies. From the 400 profiles 291 (72.7\%) are male accounts and 109 (27.3\%) are female accounts. No effort was made to verify that each account was associated with a distinct

\footnotetext{
${ }^{1}$ Shtuni, A. (2015). Breaking Down the Ethnic Albanian Foreign Fighters Phenomenon. Soundings: An Interdisciplinary Journal, 98(4), 460-477. doi:10.5325/soundings.98.4.0460

${ }^{2}$ Counter Extremism Project. 2020. Albania: Extremism \& Counter-Extremism. https://www.counterextremism.com/countries/albania [Accessed 11 August 2020].
} 
individual. For the purpose of this research, each Facebook page was considered to represent a distinct, real person.

Accounts with open support (liking or posting) for ISIS, Al-Nusra and Al-Qaeda were defined as extremist accounts and included as part of our sample. Also included were accounts of individuals supporting Jihad more broadly, but not identifying with a specific terrorist group (posting videos, photos, articles, and teachings from imams who support ISIS and other similar Jihadi organisations). Each account was thoroughly analysed and categorized based on whether the account holder "liked" or "posted" VE materials. Accounts that both liked and posted VE materials were included in the "posting" category of our data set. Out of the total amount of accounts, $49.8 \%$ were categorized as "posting" (either just posting or posting and liking) and $50.2 \%$ were categorized as "liking" (liking but not posting) Jihadi materials on Facebook. We only managed to identify a portion of such individuals since it is impossible to identify all the accounts engaging in such activities. We based our research on the information available to the public (public posts) from the sample accounts and we did not friend request, or in any way interact with, any of the subjects. Thus, we are unable to see the complete activity of the sampled profiles since we have access only to their public posts. The most common show of support was the display of insignia of various terrorist organisations, glorification of the Albanian Shahid ("martyrs") from the Syrian and Iraqi front, and supporting known regional and foreign imams with links to terrorist organisations (Rexhep Memishi, Shukri Aliu, Ebu Hanzala, etc). All the information was taken at face value, since it is hard to determine if all the profiles are of real people as opposed to virtual personas possibly created by a propaganda department within a terrorist organisation.

\section{Activity Level:}

Account activity (high, medium, low) was based on an analysis of posts spanning a one-week time frame, chronologically backwards from the day the account was identified. Accounts with at least one post a day during this timeframe were classified as highly active. Those with 4 to 6 posts were classified as medium activity. Finally, those with under 4 posts were classified as low activity. Accounts that had not posted for a week or longer were also labeled as low activity.

Keywords: ISIS, Al-Nusra, Al-Qaeda, Facebook, social media, Albania, Kosovo, North Macedonia, data collection, Jihadi, western balkans, violent extremism.

\section{Data and Results}

Female Activity

From the 109 female accounts $43.1 \%$ liked and $56.9 \%$ posted VE materials. Given that the accounts that post are most likely indoctrinated individuals, we will focus our analysis more closely on the activity of those who post.

Chart 1 shows the location and activity level of the female accounts that "post" vs "like" VE materials. Of the 109 female accounts $32.1 \%$ are from North Macedonia, 30.3\% have no location associated, 20.2\% from Kosovo, $11.9 \%$ from Albania, and 5.5\% are from other countries (Serbia, Germany and Italy). 
Accounts associated with Kosovo or "other" countries (Serbia and Italy) showed greater percentages of "high" activity, while accounts associated with Albania and North Macedonia, as well as those not associated with a location ("no location") showed comparably lower percentages of "high" activity among females.

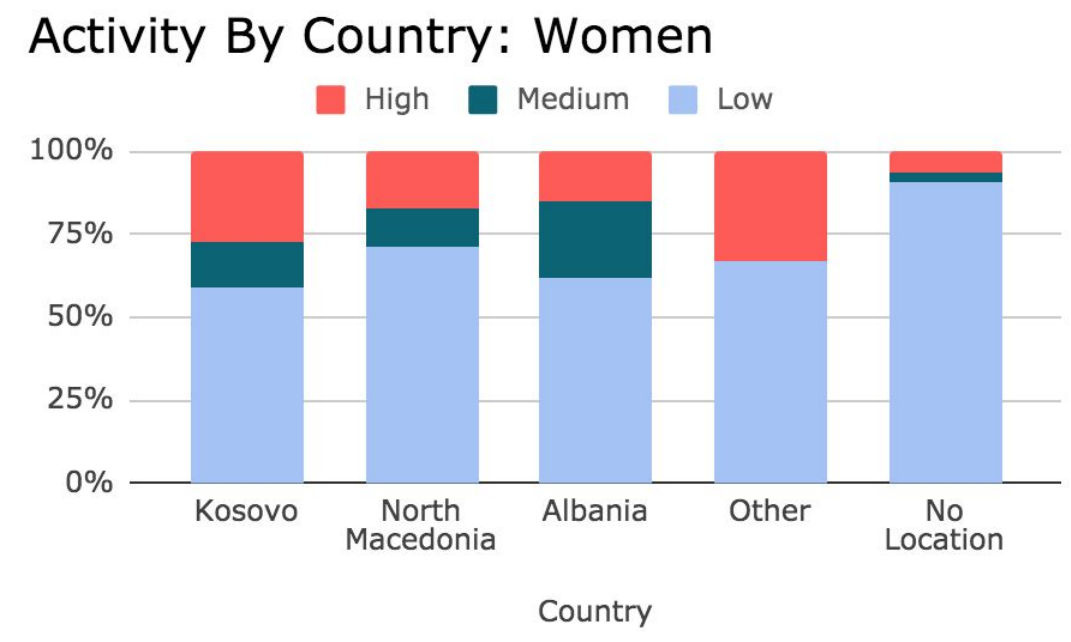

Chart 1: Activity of Women by Country

Of the female accounts, 62 were classified as "posting" accounts. Chart 2 shows the location and activity level of the female accounts that "post." Of these, $35.5 \%$ have no location associated with their profile, $33.9 \%$ are from North Macedonia, 19.4\% are from Kosovo, $8.1 \%$ are from Albania, and 3.2\% are from other countries (Serbia and Italy).

Based on our collected data, posting females in Kosovo were more likely to have a high level of activity (50\%) as compared to those from North Macedonia (19\%) or Albania (40\%).

Additionally, our data set shows that females who post from "other" countries (Serbia and Italy) have $100 \%$ high activity; however, we note that the sample size from these countries is very small and may not be representative. Finally, accounts that were not associated with a location had a comparably low level of "high" activity (9.1\%). 
回 counteradical

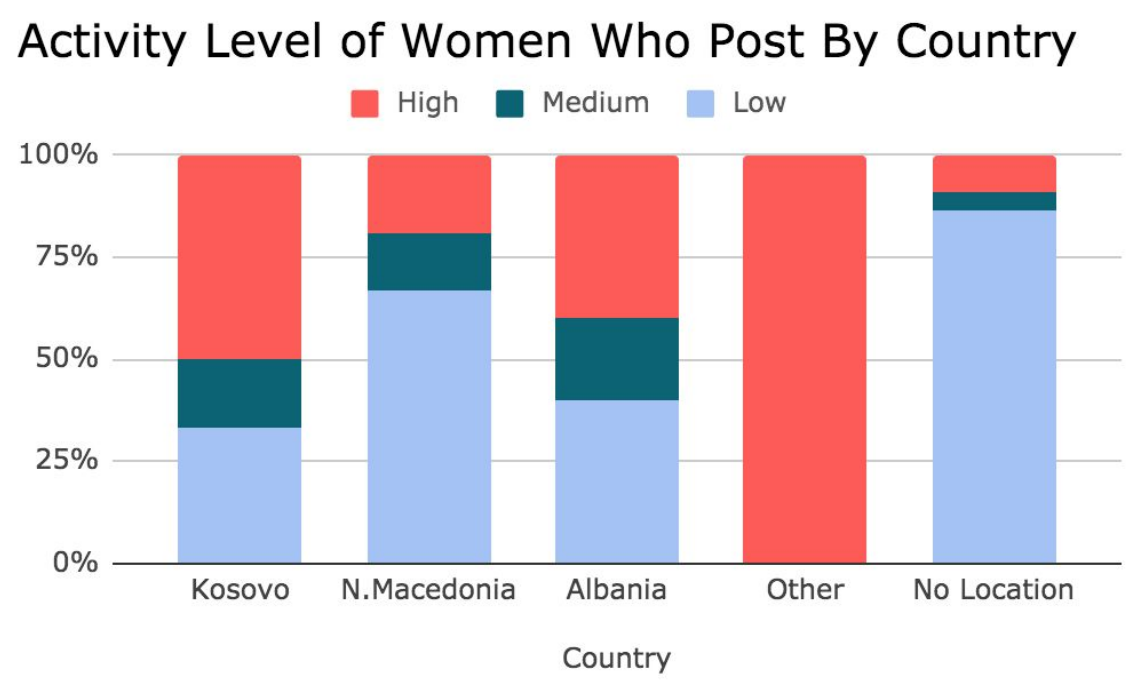

Chart 2: Activity Level of Women Who Post By Country

As noted in Chart 3, 56.9\% (62 accounts) of the total 109 female accounts were categorized as "posting." From the posting female accounts, $25.8 \%$ showed high activity, with 7 or more posts per week, $11.3 \%$ showed medium activity, and $62.9 \%$ showed low activity.

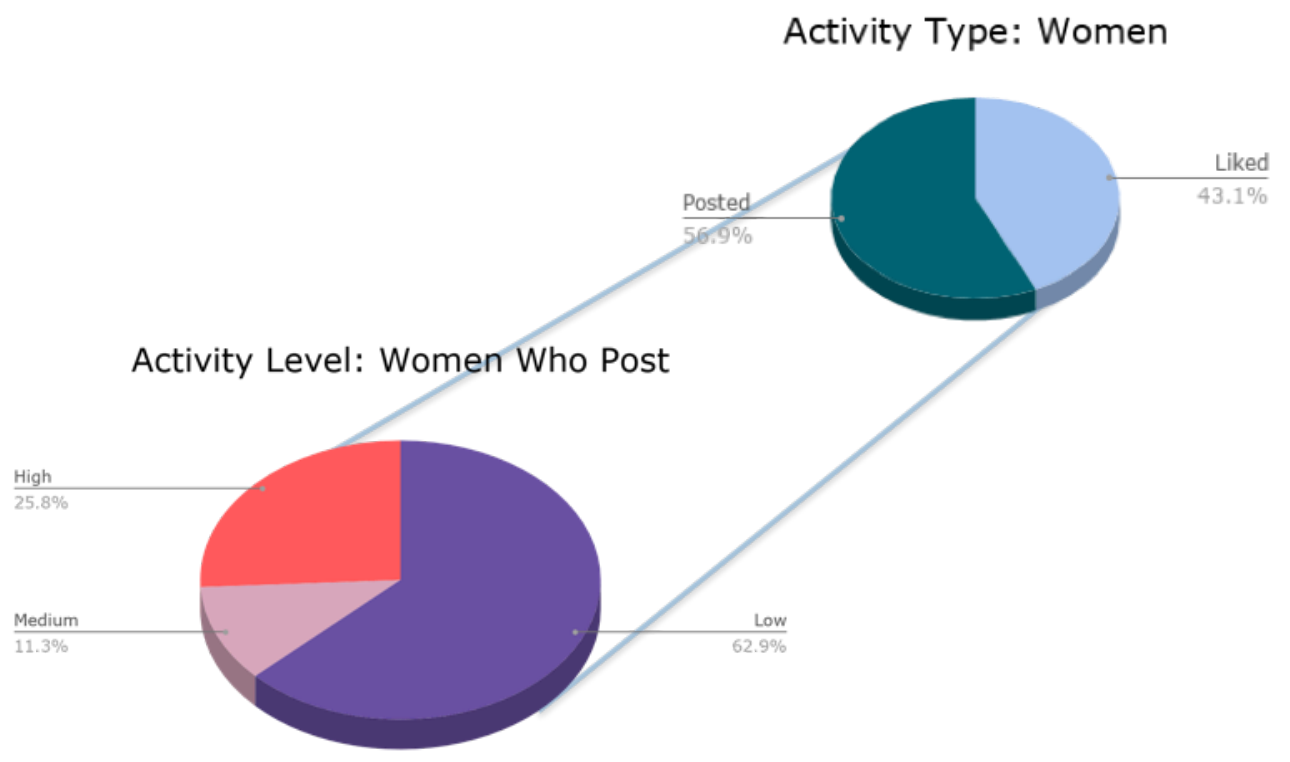

Chart 3: Female Activity

\section{Male activity}

From the 291 male accounts $47.1 \%$ posted and $52.9 \%$ liked VE materials. Given that the accounts that post are most likely indoctrinated individuals, we will focus our analysis more closely on the activity of those who post. 


\section{回 counteradical}

Of the posting accounts, $24.1 \%$ had high activity, $24.1 \%$ had medium activity, and $51.8 \%$ had low activity, as noted in Chart 4.

Activity Level: Men Who Post

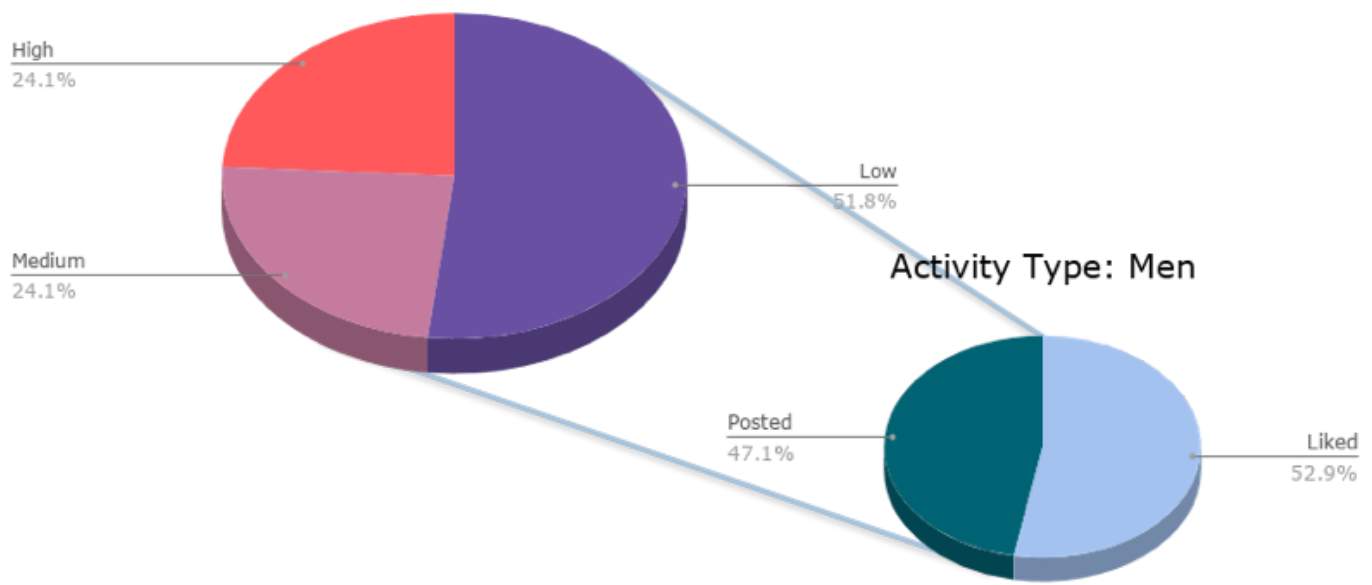

Chart 4: Male Activity

Chart 5 shows the location and activity level of the male accounts that "post" vs "like" VE materials. Of the 291 male accounts $26.5 \%$ Are from Kosovo, 26.5\% are from North Macedonia, $11 \%$ from Albania, 6.2\% are from other countries (Serbia, Germany, Sweden, Turkey, USA, Syria, Greece, Italy, Saudi Arabia, France), and 30.7\% have no location.

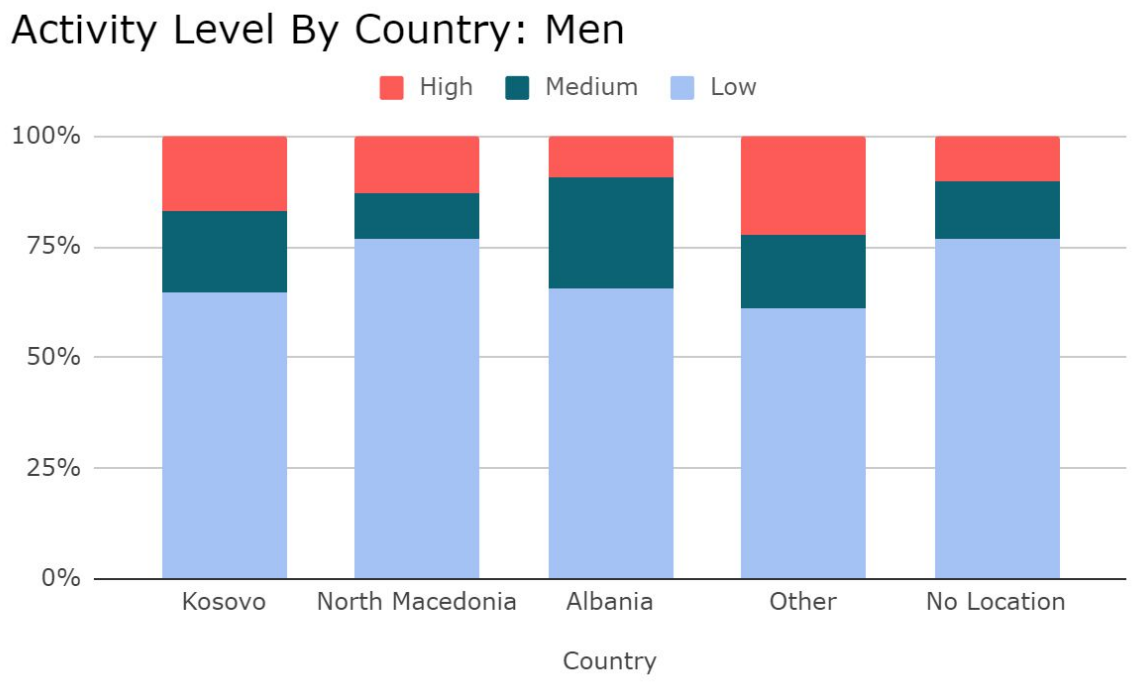

Chart 5: Activity of Men by Country 
Of the male accounts, 137 were classified as "posting" accounts. Chart 6 shows the location and activity level of the male accounts that "post". Of these, $30.7 \%$ have no location associated with their profile, 27\% are from North Macedonia, 26.3\% are from Kosovo, 9.5\% are from Albania, and $6.6 \%$ are from other countries (Serbia, Germany, Sweden, Turkey, USA and Syria).

Based on our collected data, posting males in Kosovo were more likely to have a higher level of activity (30.6\%) as compared to those from North Macedonia (24.3\%) and Albania (23.1\%). Additionally, our data set shows that females who post from "other" countries (Serbia, Germany, Sweden, Turkey, USA and Syria) have 33.3\% high activity but the sample size from these countries is small and may not be representative. Finally, accounts that were not associated with a location had a comparably low level of "high" activity (16.7\%).

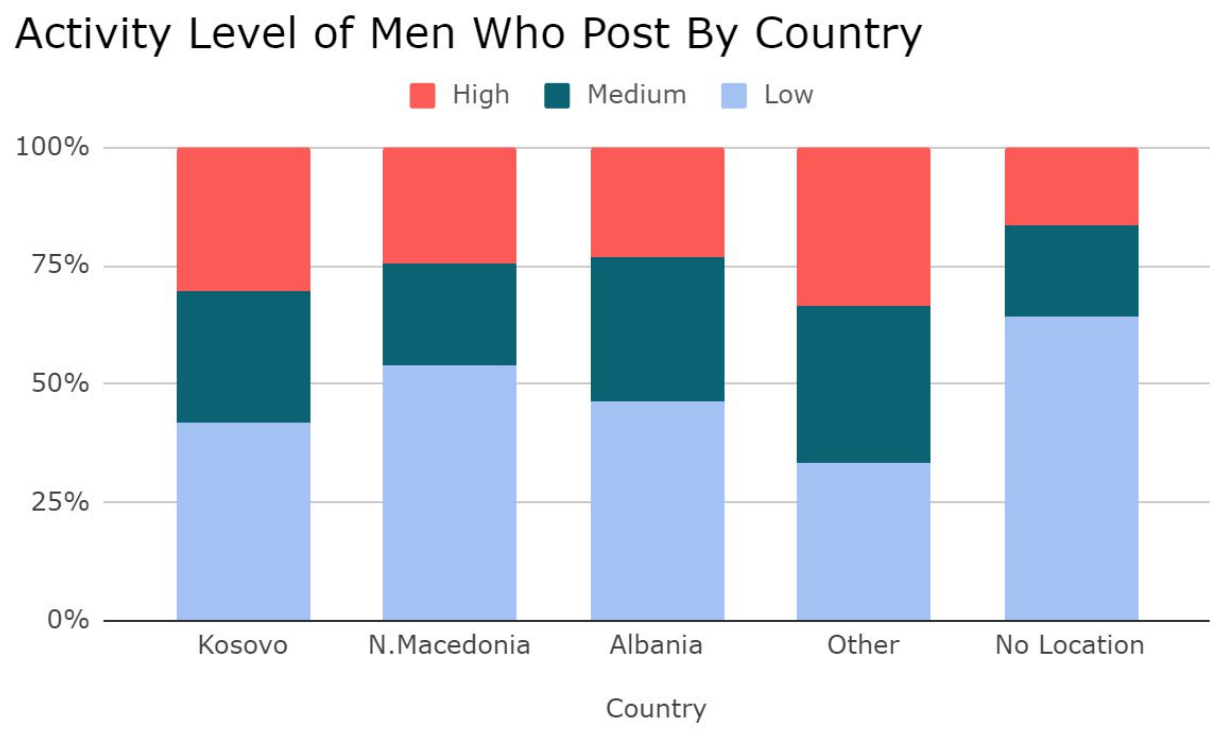

Chart 6: Activity Level of Men Who Post By Country

\section{Conclusion}

This research intends to break down activity by gender and location for Albanian speaking Jihadis on Facebook. The results of our study show that terrorist organisations continue to maintain their social media presence through individual accounts that spread VE content. Given the lack of statistical error analysis in this study, it is difficult to make definitive numerical comparisons between male and female activity. Nevertheless, we are able to draw certain conclusions from the data. Specifically, it is apparent that both male and female individuals are actively promoting VE content on social media. Additionally, within both male and female groups, we observe varying activity levels, and activity types ranging from only "liking" VE content to actively posting and sharing the content on a regular basis. Also, all three countries (Kosovo, North Macedonia, and Albania) present a relatively equal proportion of Jihadi individuals who post VE materials on Facebook. 
Our primary focus was on individuals who post and share Jihadi content, as these are more likely to be indoctrinated into the ideology. Among this group, there is a higher percentage of "high activity" accounts when compared to the "liking" category. It is very likely that these accounts are trying to radicalize people in their circles so they can increase their support base or maintain an online presence. Thus, we draw the conclusion that individuals who post or share VE content are more likely to be highly active.

Additionally, we also discovered numerous Albanian-speaking accounts located outside of Albanian-speaking countries and countries with significant Albanian populations. Countries that make the "other" location category include Serbia, Germany, Sweden, Turkey, Syria, USA, Italy, Saudi Arabia, Greece, France, and Czechia). Thus, we can infer from this information that Albanian-language VE content reaches beyond the predominantly Albanian-speaking region of the Balkans. As noted above, these accounts showed higher percentages of "high" activity levels. This may be because of immigrant communities failing to integrate into the societies they live in.

Even though female accounts are present on Facebook, they present a challenge since it is difficult to view their profiles and measure activity. Most of the Jihadi female accounts continue to vet their friend requests and only accept acquainted individuals who are mostly other female friends or family members. As a result, we were able to identify significantly fewer female accounts than male. Nevertheless, it is clear that female account holders are also active in spreading VE content both in the Balkans, and in other parts of the world. Among these individuals, numerous accounts showed "high" activity levels comparable to that of their male counterparts.

\section{Future studies:}

To expand on this study it could be useful to research and monitor individuals who post and share VE content in order to determine what motivates individuals to increase their activity (low to high). Additionally, a significant amount of people are being targeted on social media, thus, a future study could focus on how and when individuals switch from only liking VE materials to posting/sharing. Equally important would be analysing accounts from the diaspora and identifying the source of their radicalisation.

\section{References:}

Shtuni, A. (2015). Breaking Down the Ethnic Albanian Foreign Fighters Phenomenon. Soundings: An Interdisciplinary Journal, 98(4), 460-477. doi:10.5325/soundings.98.4.0460

Counter Extremism Project. 2020. Albania: Extremism \& Counter-Extremism. https://www.counterextremism.com/countries/albania [Accessed 11 August 2020]. 